\title{
The Effect of Spatially Dependent Temperature and Carrier Fluctuations on Noise in Semiconductor Lasers
}

\author{
ROBERT J. LANG, STUDENT MEMBER, IEEE, KERRY J. VAHALA, STUDENT MEMBER, IEEE, \\ AND AMNON YARIV, FELLOW, IEEE
}

\begin{abstract}
The spatially dependent equations of motion for a singlemode semiconductor laser including Langevin source terms are derived and solved. The relative intensity, frequency, and field fluctuation spectra are derived and calculated. The results include low-frequency excess noise, frequency noise enhancement due to two forms of amplitudephase coupling, and power-independent contributions to the linewidth.
\end{abstract}

\section{INTRODUCTION}

$\mathrm{O}$ VER the past twenty years, semiconductor lasers have evolved into a reliable source of coherent near-IR light. Their small size, low power requirement, and ability to be directly modulated at microwave frequencies has, in more recent years, made them the subject of intense study for use in communications systems, particularly fiber optics transmission systems. Part of this interest has been directed towards characterizing their noise properties and the consequent effects on transmission of information. Several anomalous features in their noise spectra have been observed, including a spiking resonance in the intensity spectrum [1] and the frequency fluctuation spectrum [4], a linewidth some thirty times greater than that predicted by the modified Schawlow-Townes theory [2], power-independent linewidth components [3], [25], assymmetry in the field spectrum [4], and excess noise at low frequencies in both the intensity and frequency fluctuation spectrum [5], [6], [7], [25]. Several theoretical models, both semiclassical and quantum mechanical, have explained many of these features. Early work by McCumber [10] explained the spiking resonance in the intensity spectrum as a result of photon-inversion dynamics using a Langevin approach; treatments by Lax [8] and Haug and Haken [17] also noted this effect and in addition provided a quantum-mechanical treatment. More recently, gain spectrum detuning has been invoked by several authors to explain linewidth enhancement, the assymmetrical field spectrum, and resonance structure in the frequency fluctuation spectrum [9], [15]. Other theoretical and experimental works have corroborated these results [1], [11]. To date, however, there has been no satisfactory explanation of the observed rise in both intensity and frequency noise below approximately $1 \mathrm{MHz}$.

Most theories either assume a priori or quickly reduce to spatially independent sets of equations. This approach offers

Manuscript received October 8, 1984; revised January 23, 1985. This work was supported in part by the Office of Naval Research, by the ITT Corporation, and by Rockwell International. R. J. Lang was supported in part by a fellowship from the National Science Foundation. $\mathrm{K}$. J. Vahala was supported in part by a fellowship from IBM.

The authors are with the California Institute of Technology, Pasadena, CA 91125. the distinct advantage of reducing a multidimensional set of partial differential equations to a one-dimensional set of ordinary differential equations, and the assumption's validity in justified by the large number of effects predicted or explained by such theories. Still, there are features of the laser dynamics that are lost; implicit in such treatments are the assumptions that averages of products are equal to the product of the averages, and any fluctuations in spatial distributions are lost in the averaging process [12]

Diffusion, for example, plays no role in a spatially independent treatment, yet diffusion both damps out spatial tluctuations and introduces fluctuations of its own. Voss and Clarke [13] and van Vliet and Mehta [14] have proposed diffusion noise as a major source of low-frequency transport noise in semiconductors. Using a Langevin approach, they calculated carrier and temperature fluctuations in different geometries, and from those, current and voltage noise.

The analysis of a semiconductor laser is somewhat different from that of the bulk semiconductor, however, because of the additional coupling between carriers, temperature distribution, and the optical field. In this paper, we shall solve the spatially varying equations of motion for the carrier density, temperature distribution, and lasing field. The results of the analysis will show that spatially varying temperature fluctuations contribute significantly to the low-frequency noise in both the intensity and frequency fluctuation spectra, and that diffusion controls the precise structure of the spectra. Carrier fluctuations, while present, are shown to contribute minimally to the two spectra. In Section II, we set up transport equations for the photon field, carrier density, and temperature distribution with Langevin sources; we linearize them, and transform the small-signal equations to spatial and temporal frequency-space. In Section III, we normalize the Langevin sources to the dy. namical variables and relate them to the macroscopic transition rates in the laser. In Section IV, we derive expressions for relative intensity, frequency, and field fluctuation spectra, including simplified results for the low-frequency limit; we present numerical examples of the results, and compare them with experimental observations. We conclude in Section $\mathrm{V}$ by summarizing several important results of the analysis.

\section{EQuations of Motion}

\section{A. Transport Equations}

The starting point for the analysis is the set of equations describing the time evolution of the carrier density, temperature, and laser mode amplitude. The equations are, respectively, for 
carriers

$$
\dot{N}=D_{E} \nabla^{2} N-\frac{\epsilon_{0}}{2 \hbar} \chi_{i}(N, T)\left|E_{n}\right|^{2}-R_{s}(N)+R(\boldsymbol{x})+\Xi
$$

where $N(\boldsymbol{x})$ is the carrier density, $D_{E}$ is the electronic diffusivity, $R_{s}$ is the spontaneous emission rate per unit volume, $R(\boldsymbol{x})$ is the pump rate, $\Xi$ is a Langevin source, and $\chi_{i}(N, T)$ is the imaginary part of the susceptibility. For temperature

$$
\dot{T}=D_{T} \nabla^{2} T+\Theta
$$

where $T(\boldsymbol{x})$ is the temperature, $D_{T}$ is the thermal diffusivity, and $\Theta$ is a Langevin source. For the electric field, the short (picosecond) round-trip time of the cavity lets one treat the lasing mode as effectively seeing all parts of the cavity at once, so that the optical field responds to integrals over the lasing volume. The field equation is [15]

$$
\ddot{E}_{n}+\frac{1}{\tau_{p}} \dot{E}_{n}+\omega_{n}^{2} E_{n}=\frac{-1}{\epsilon_{0} \mu^{2} V} \int d^{3} \boldsymbol{x} \ddot{\boldsymbol{P}} \cdot \boldsymbol{e}_{n}(\boldsymbol{x})+\Delta e^{j \omega_{m} t}
$$

where $E_{n}$ is the electric field amplitude of the $n$th mode, $\omega_{n}$ is the $n$th resonant frequency of the unpumped cavity, $\tau_{p}$ is the photon lifetime, $\mu$ is the nonresonant contribution to refractive index, $\boldsymbol{P}(\boldsymbol{x})$ is the induced polarization, $\Delta$ is a Langevin source, $\omega_{m}$ is the actual lasing frequency, and $\left\{\boldsymbol{e}_{n}(\boldsymbol{x})\right\}$ are the normalized $n$th spatial modes of the unpumped resonator, where

$$
\int d^{3} \boldsymbol{x}\left|\boldsymbol{e}_{n}(\boldsymbol{x})\right|^{2}=V
$$

and $V$ is the volume of the lasing mode.

These equations are then linearized about operating points

$$
\begin{aligned}
N(\boldsymbol{x}) & \equiv N_{0}(\boldsymbol{x})+\nu(\boldsymbol{x}, t) \\
R_{s}(N) & \equiv R_{s}\left(N_{0}\right)+\omega_{s} \cdot \nu \\
E_{n}(t) & \equiv A_{0}(1+\rho(t)) e^{j\left(\omega_{m} t+\varphi(t)\right)} \\
T(\boldsymbol{x}) & \equiv T_{0}(\boldsymbol{x})+\tau(\boldsymbol{x}, t) \\
\chi(N, T) & \approx \chi\left(N_{0}, T_{0}\right)+\zeta \nu+\eta \tau
\end{aligned}
$$

where

$$
\left.\zeta \equiv \frac{\partial \chi}{\partial N}\right|_{N=N_{0}, T=T_{0}},\left.\eta \equiv \frac{\partial \chi}{\partial T}\right|_{N=N_{0}, T=T_{0}}
$$

and

$$
\left.\omega_{s} \equiv \frac{\partial R_{s}}{\partial N}\right|_{N=N_{0}, T=T_{0}}
$$

is the differential spontaneous emission rate per unit volume. If the laser is assumed to be running in a single mode, then

$$
P(\boldsymbol{x})=\epsilon_{0} \chi(N, T) \boldsymbol{E}(\boldsymbol{x})=\epsilon_{0} \chi(N, T) E_{n} \boldsymbol{e}_{n}(\boldsymbol{x})
$$

so that

$$
\begin{aligned}
& \frac{1}{\epsilon_{0} \mu^{2} V} \int d^{3} \boldsymbol{x} \ddot{\boldsymbol{P}}(\boldsymbol{x}) \cdot \boldsymbol{e}_{n}(\boldsymbol{x}) \\
& \quad \approx\left[\frac{1}{\epsilon_{0} \mu^{2} V} \int d^{3} \boldsymbol{x} \epsilon_{0}\left(\chi\left(N_{0}, T_{0}\right)+\zeta \nu+\eta \tau\right) E_{n}\left|\boldsymbol{e}_{n}(\boldsymbol{x})\right|^{2}\right]^{\cdot \cdot}
\end{aligned}
$$

We can define an "effective susceptibility" as

$$
\chi_{\Gamma} \equiv \frac{1}{V} \int_{V} d^{3} \boldsymbol{x} \chi\left(N_{0}, T_{0}\right)\left|\boldsymbol{e}_{n}(\boldsymbol{x})\right|^{2} .
$$

Substitute definitions (4) -(6) into (1)-(3), neglecting terms of second order or less to get

$$
\begin{aligned}
& \dot{\nu}= D_{E} \nabla^{2} N_{0}+D_{E} \nabla^{2} \nu-\frac{\epsilon_{0}}{2 \hbar}\left[\chi_{i}\left(N_{0}\right) A_{0}^{2}(1+2 \rho)+\zeta_{i} \nu A_{0}^{2}\right] \\
&-R_{s}\left(N_{0}\right)-\omega_{s} \nu+R+\Xi \\
& \dot{\tau}= D_{T} \nabla^{2} T_{0}+D_{T} \nabla^{2} \tau+\Theta \\
& 2 j \omega_{m} A_{0}(\dot{\rho}+j \dot{\varphi})-\frac{\omega_{m}^{2} A_{0}}{\mu^{2} V} \int_{V} d^{3} \boldsymbol{x}(\zeta \nu+\eta \tau)\left|\boldsymbol{e}_{n}\right|^{2} \\
&+\left(\omega_{n}^{2}-\omega_{m}^{2}+j \frac{\omega_{m}}{\tau_{p}}-\frac{\omega_{m}^{2}}{\mu^{2}} \chi_{\Gamma}\right) A_{0}=\Delta e^{-j \varphi(t)}
\end{aligned}
$$

Here $r$ and $i$ subscripts on $\zeta, \eta, \chi$, and $\Delta$ denote the real and imaginary parts, respectively, of the quantities which bear them.

The zeroth-order terms in (7)-(9) establish the operating point for the laser; the first-order terms describe the smallsignal response to the Langevin driving terms. Since (1) we are only interested in the second moment of $\Delta$, and (2) $\Delta$ is approximately white over the laser line, we can drop the $e^{-j \varphi}$ on the right hand side of (9); separating real and imaginary parts of (9) and taking out the zeroth order terms gives

$$
\begin{aligned}
\dot{\rho}-\frac{\omega_{m}}{2 \mu^{2} V} \int d^{3} \boldsymbol{x}\left(\zeta_{i} \nu+\eta_{i} \tau\right)\left|\boldsymbol{e}_{n}\right|^{2} & =\frac{1}{2 \omega_{m} A_{0}} \Delta_{i} \\
\dot{\varphi}+\frac{\omega_{m}}{2 \mu^{2} V} \int d^{3} \boldsymbol{x}\left(\zeta_{r} \nu+\eta_{r} \tau\right)\left|\boldsymbol{e}_{n}\right|^{2} & =\frac{-1}{2 \omega_{m} A_{0}} \Delta_{r} \\
\dot{\nu}-D_{E} \nabla^{2} \nu+\frac{1}{\tau_{R}} \nu+\frac{\epsilon_{0} \chi_{i}\left(N_{0}, T_{0}\right) A_{0}^{2}}{h} \rho & =\Xi \\
\dot{\tau}-D_{T} \nabla^{2} \tau & =\Theta
\end{aligned}
$$

where $1 / \tau_{R} \equiv \omega_{s}+\epsilon_{0} A_{0}^{2} \zeta_{i}(\boldsymbol{x}) / 2 \hbar$. Equations (10)-(13) comprise the fluctuation equations describing the small-signal behavior of the relative amplitude $(\rho(t))$, phase $(\varphi(t))$, carrier density $(\nu(\boldsymbol{x}, t))$, and temperature $(\tau(\boldsymbol{x}, t))$ of a single-mode semiconductor laser.

\section{B. Fourier Analysis}

Equations (12) and (13) are partial differential equations involving spatial derivatives in the Laplacian operator. An 
exact solution must take into account the spatial variation of $1 / \tau_{R}$ and the boundary conditions, the latter of which are in general mixed and/or inhomogeneous. Unfortunately, configurations for which closed-form solutions exist are few and far between. We will make three simplifying assumptions.

1) $\zeta_{i}, \zeta_{r}$, and $\omega_{s}$ (and thus $1 / \tau_{R}$ ) are constant over the active region.

2) The carriers are confined to the active region so that the normal derivative of $\nu$ vanishes on the boundary (e.g., an ideal buried heterostructure). We can include structures with no lateral confinement (e.g., gain-guided) by allowing the lateral confinement direction to approach infinity.

3) The temperature profile is subject to fictitious boundary conditions; all functions and derivatives are continuous across the boundary of the device (i.e., we neglect variations in material parameters resulting from compositional shifts from GaAs to $\mathrm{AlGaAs}$ ).

We now define Fourier transforms. For quantities which vary in time

$$
\tilde{f}(\omega) \equiv \int d t f(t) e^{-j \omega t}, \quad f(t)=\frac{1}{2 \pi} \int d \omega \tilde{f}(\omega) e^{j \omega t} .
$$

For quantities which vary in space and have fictitious boundary conditions

$$
\begin{aligned}
& \tilde{g}(\boldsymbol{k}) \equiv \int d^{3} \boldsymbol{x} g(\boldsymbol{x}) e^{j \boldsymbol{k} \cdot \boldsymbol{x}}, \\
& g(\boldsymbol{x})=\frac{1}{(2 \pi)^{3}} \int d^{3} \boldsymbol{k} \tilde{g}(\boldsymbol{k}) e^{-j \boldsymbol{k} \cdot \boldsymbol{x}} .
\end{aligned}
$$

For carrier fluctuations confined to a region $S_{1} \times S_{2} \times S_{3}$ with volume $V_{A}=S_{1} S_{2} S_{3}$, the finite domain gives rise to a discrete spectrum with eigenfunctions defined as

We make use of the identity

$$
\int d^{3} \boldsymbol{x} f(\boldsymbol{x}) g(\boldsymbol{x})=\frac{1}{(2 \pi)^{3}} \int d^{3} \boldsymbol{k} \tilde{f}(\boldsymbol{k}) \tilde{g}(-\boldsymbol{k})
$$

define

$$
\Gamma(\boldsymbol{k}) \equiv \frac{1}{(2 \pi)^{3}} \int d^{3} \boldsymbol{x}\left|\boldsymbol{e}_{n}(\boldsymbol{x})\right|^{2} e^{-j \boldsymbol{k} \cdot \boldsymbol{x}}
$$

and approximate

$$
\tilde{\eta}_{i, r}(\boldsymbol{k}) \approx \bar{\eta}_{i, r} \cdot(2 \pi)^{3} \Gamma(\boldsymbol{k})
$$

(which is tantamount to assuming that $\eta$ is constant over the optical mode) so that the fluctuation equations, transformed in both space and time, become

$$
\begin{aligned}
& j \omega \tilde{\rho}(\omega)-\frac{\omega_{m}}{2 \mu^{2} V} \int d^{3} \boldsymbol{x}\left|\boldsymbol{e}_{n}(\boldsymbol{x})\right|^{2} \zeta_{i} \sum_{n} \frac{\gamma(\boldsymbol{n})}{V_{A}} \tilde{\nu}(\boldsymbol{n}, \omega) g(\boldsymbol{n}, \boldsymbol{x}) \\
& \quad-\frac{\omega_{m}}{2 \mu^{2} V} \int d^{3} \boldsymbol{k} \bar{\eta}_{i} \Gamma(\boldsymbol{k}) \tilde{\tau}(\boldsymbol{k}, \omega)=\frac{1}{2 \omega_{m} A_{0}} \tilde{\Delta}_{i}(\omega) \\
& j \omega \tilde{\varphi}(\omega)+\frac{\omega_{m}}{2 \mu^{2} V} \int d^{3} \boldsymbol{x}\left|\boldsymbol{e}_{n}(\boldsymbol{x})\right|^{2} \zeta_{r} \sum_{n} \frac{\gamma(\boldsymbol{n})}{V_{A}} \tilde{\nu}(\boldsymbol{n}, \omega) g(\boldsymbol{n}, \boldsymbol{x}) \\
& \quad+\frac{\omega_{m}}{2 \mu^{2} V} \int d^{3} \boldsymbol{k} \bar{\eta}_{r} \Gamma(\boldsymbol{k}) \tilde{\tau}(\boldsymbol{k}, \omega)=\frac{-1}{2 \omega_{m} A_{0}} \tilde{\Delta}_{r}(\omega) \\
& \left(j \omega+D_{E} q^{2}(\boldsymbol{n})+\frac{1}{\tau_{R}}\right) \tilde{\nu}(\boldsymbol{n}, \omega)+\frac{\epsilon_{0} \tilde{\chi}_{i}(\boldsymbol{n}) A_{0}^{2}}{\hbar} \tilde{\rho}(\omega) \\
& \quad=\tilde{\Xi}(\boldsymbol{n}, \omega) \\
& \left(j \omega+D_{T^{2}} k^{2}\right) \tilde{\tau}(\boldsymbol{k}, \omega)=\widetilde{\Theta}(\boldsymbol{k}, \omega) .
\end{aligned}
$$

$$
g(\boldsymbol{n}, \boldsymbol{x}) \equiv \begin{cases}\cos q_{1}\left(x_{1}-\frac{S_{1}}{2}\right) \cos q_{2}\left(x_{2}-\frac{S_{2}}{2}\right) \cos q_{3}\left(x_{3}-\frac{S_{3}}{2}\right) & \text { for } x_{i} \in\left[\frac{-S_{i}}{2}, \frac{S_{i}}{2}\right] \\ 0 & \text { otherwise }\end{cases}
$$

having index vector $\boldsymbol{n}$; the wavevector $\boldsymbol{q}(\boldsymbol{n})$ is defined by

$$
q_{i}(\boldsymbol{n}) \equiv \frac{n_{i} \pi}{S_{i}} .
$$

The associated spectral representation is

$$
\begin{aligned}
& \tilde{h}(\boldsymbol{n}) \equiv \int_{V_{A}} d^{3} \boldsymbol{x} h(\boldsymbol{x}) g(\boldsymbol{n}, \boldsymbol{x}), \\
& h(\boldsymbol{x})=\sum_{\boldsymbol{n}} \frac{\gamma(\boldsymbol{n})}{V_{A}} \tilde{h}(\boldsymbol{n}) g(\boldsymbol{n}, \boldsymbol{x}) .
\end{aligned}
$$

In this expression, the $\left\{n_{i}\right\}$ can take on integer values from 0 to $\infty$, and $\gamma(\boldsymbol{n})$ is a normalization factor equal to $1,2,4$, or 8 depending on how many of the $n_{i}$ 's are zero $(0,1,2$, or 3 , respectively). Then the operators in (10)-(13) transform as

$$
\frac{\partial}{\partial t} \rightarrow j \omega, \quad \nabla^{2} \rightarrow-k^{2} \text { or }-q^{2}(\boldsymbol{n}) .
$$

Elimination of carrier density $(\tilde{\nu})$ and temperature distribution ( $\tilde{\tau})$ from (22)-(25) gives

$$
\begin{aligned}
\tilde{\rho}(\omega)= & R(\omega)\left[\frac{\omega_{m}}{2 \mu^{2} V} \sum_{\boldsymbol{n}} \Lambda(\boldsymbol{n}) \zeta_{i} \frac{\tilde{\Xi}(\boldsymbol{n}, \omega)}{j \omega+D_{E} q^{2}(\boldsymbol{n})+\frac{1}{\tau_{R}}}\right. \\
& \left.+\frac{\omega_{m}}{2 \mu^{2} V} \int d^{3} \boldsymbol{k} \Gamma(\boldsymbol{k}) \bar{\eta}_{i} \frac{\tilde{\Theta}}{j \omega+D_{T} k^{2}}+\frac{1}{2 \omega_{m} A_{0}} \tilde{\Delta}_{i}\right] \\
j \omega \tilde{\varphi}(\omega)= & {\left[\frac{\zeta_{r}}{\zeta_{i}}(1-j \omega R)-\frac{\bar{\eta}_{r}}{\left.\bar{\eta}_{i}\right] \int d^{3} \boldsymbol{k} \Gamma(\boldsymbol{k}) \bar{\eta}_{i} \frac{\Theta(\boldsymbol{k}, \omega)}{j \omega+D_{T} k^{2}}}\right.} \\
& -j \omega R \frac{\tilde{\Xi}(\boldsymbol{n}, \omega)}{\zeta_{i}} \sum_{\boldsymbol{n}} \Lambda(\boldsymbol{n}) \zeta_{i}-j \omega+D_{E} q^{2}(\boldsymbol{n})+\frac{1}{\tau_{R}} \\
& -\frac{1}{2 \omega_{m} A_{0}} \tilde{\Delta}_{r}+\frac{\zeta_{r}}{\zeta_{i}}(1-j \omega R) \frac{1}{2 \omega_{m} A_{0}} \tilde{\Delta}_{i}
\end{aligned}
$$




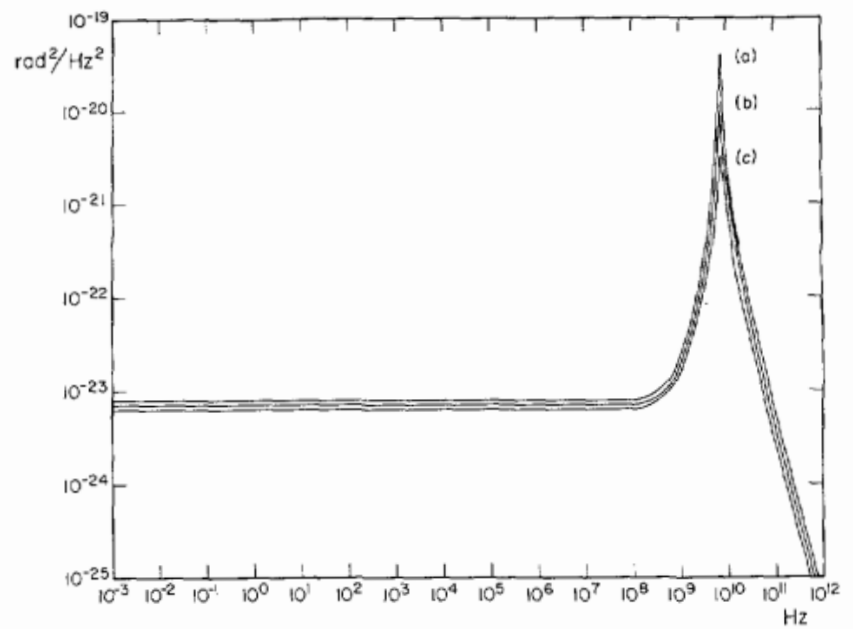

Fig. 1. Effect of inhomogeneities in carrier density and optical field on relaxation resonance $|R(\Omega)|^{2}$ in the intensity noise spectrum. The optical field distribution is taken to be $\frac{1}{2}\left(1+\cos \left(2 \pi x_{i} / S_{i}\right)\right)$ in the lateral direction and susceptibility is taken to be proportional to $[1 /(1+\epsilon)]\left[1+\epsilon \cos \left(2 \pi x_{i} / S_{i}\right)\right]$ in the lateral direction. Transverse variation contributes negligibly to the resonance, so for a symmetric structure, the dominant damping comes from the $\boldsymbol{n}=(0,2,0)$ term. (a) $\epsilon=0$. (b) $\epsilon=0.5, S_{2}=2.0 \mu \mathrm{m}$. (c) $\epsilon=0.5, S_{2}=4.0 \mu \mathrm{m}$.

where

$$
\begin{aligned}
& \Lambda(\boldsymbol{n}) \equiv \frac{\gamma(\boldsymbol{n})}{V_{A}} \int d^{3} \boldsymbol{x}\left|\boldsymbol{e}_{n}(\boldsymbol{x})\right|^{2} g(\boldsymbol{n}, \boldsymbol{x}) \\
& \frac{1}{R(\omega)} \equiv j \omega+\sum_{\boldsymbol{n}} \frac{\omega_{m} \zeta_{i} \epsilon_{0} \tilde{\chi}_{i}(\boldsymbol{n}) \Lambda(\boldsymbol{n}) A_{0}^{2}}{2 \mu^{2} \grave{h} V} \\
& \cdot\left[j \omega+D_{E} q^{2}(\boldsymbol{n})+\frac{1}{\tau_{R}}\right]^{-1} .
\end{aligned}
$$

\section{Relaxation Resonance}

If either $\chi_{i}(\boldsymbol{x})$ or $\left|\boldsymbol{e}_{n}(\boldsymbol{x})\right|$ are constant over the active region then the only nonzero $\tilde{\chi}_{i}(\boldsymbol{n})$ or $\Lambda(\boldsymbol{n})$ is the $\boldsymbol{n}=(0,0,0)$ term. In this situation, $R(\omega)$ reduces to McCumber's relaxation resonance [10]

$$
R(\omega)=\frac{j \omega+\frac{1}{\tau_{R}}}{\omega_{R}^{2}-\omega^{2}+\frac{j \omega}{\tau_{R}}}, \quad \omega_{R}^{2} \equiv \frac{\omega_{m} \zeta_{i} \epsilon_{0} \chi_{i} \Lambda A_{0}^{2}}{2 \mu^{2} \hbar}
$$

where $\Lambda$ is the fill factor. However, if both $\chi_{i}(\boldsymbol{x})$ and $\left|\boldsymbol{e}_{n}(\boldsymbol{x})\right|$ vary over the active region, then there are nonzero values for $\tilde{\chi}_{i}(\boldsymbol{n})$ and $\Lambda(\boldsymbol{n})$, which contribute additional terms to the resonance expression. Physically, this is a demonstration of the fact that there is a considerable diffusive flow of carriers if the carrier density is not uniform, and the phase lag between the diffusion process and the normal carrier-photon dynamics alters the frequency response [23]. For typical dimensions and distributions, the result is a damping of both the intensity resonance $|R(\omega)|^{2}$ and the frequency resonance $|1-j \omega R(\omega)|^{2}$. Figs. 1 and 2 illustrate the damping for several different configurations. It is interesting to note that if any of the products $\tilde{\chi}_{i}(\boldsymbol{n}) \Lambda(\boldsymbol{n})$ are negative, they can have the effect of sharpening the resonance; however, such terms decrease the overlap between the gain and the optical mode, degrading the perfor-

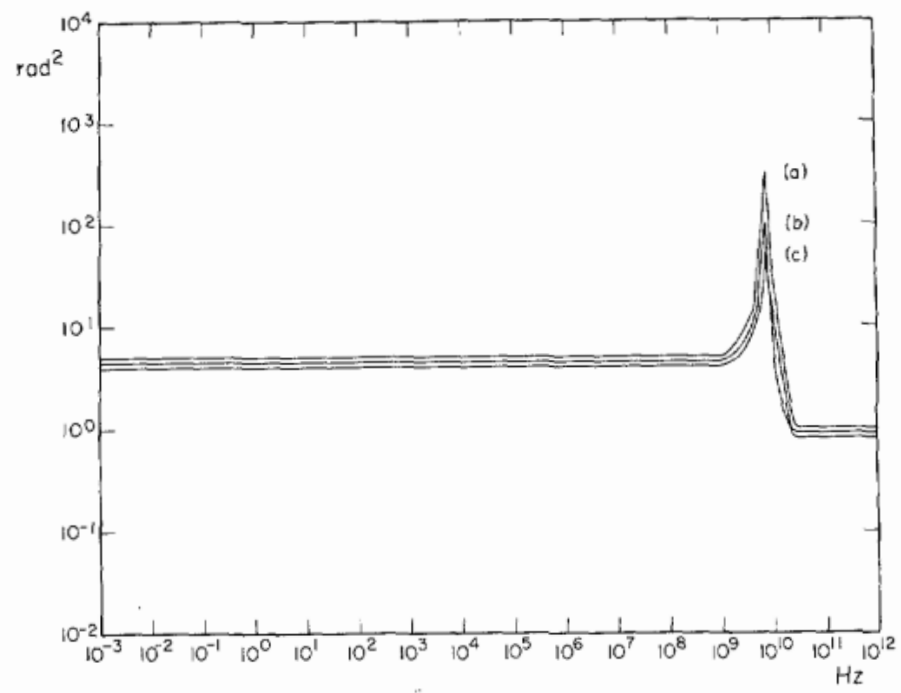

Fig. 2. Same parameters for frequency fluctuation resonance $1+$ $\alpha_{N}^{2}|1-j \Omega R|^{2}$.

mance of the laser and making it unlikely that such a mode would be the preferred mode of oscillation.

\section{LANGEVIN SOURCES}

\section{A. Rate Normalization}

When several systems of particles interact with each other and/or with external baths through random particle interactions, there are fluctuations associated with each interaction. Such fluctuations can be accounted for by including appropriately normalized Langevin sources into the equations of motion. This approach can be used with systems which vary continuously (e.g., temperature) if the interaction being characterized by a Langevin source is particulate (that is, the interaction occurs in discrete units, such as photon absorption or nonradiative recombination). Each independent number variable $\{A\}$ will have associated with it a fluctuation source $\{a\}$ which satisfies

$$
\left\langle a(t) a\left(t^{\prime}\right)\right\rangle=\left\langle\frac{d A}{d t}\right\rangle \delta\left(t-t^{\prime}\right)
$$

where \langle\rangle denotes ensemble average. For a number variable which varies in space as well, one has the modified relation

$$
\left\langle a(\boldsymbol{x}, t) a\left(\boldsymbol{x}^{\prime}, t^{\prime}\right)\right\rangle=\left\langle\frac{d A(\boldsymbol{x}, t)}{d t}\right\rangle \delta\left(t-t^{\prime}\right) \delta^{3}\left(\boldsymbol{x}-\boldsymbol{x}^{\prime}\right) .
$$

There are additional fluctuations associated with the microscopic kinetics of carrier and thermal diffusion, however. These have a different spatial correlation than the previous sources. Van Vliet and Mehta [41] have shown that the sources obey

$$
\begin{aligned}
& \left\langle\xi_{D}(\boldsymbol{x}, t) \xi_{D}\left(\boldsymbol{x}^{\prime}, t^{\prime}\right)\right\rangle \\
& \quad=2 D_{E} \nabla_{\boldsymbol{x}} \cdot \nabla_{\boldsymbol{x}^{\prime}}\left[N_{0}(\boldsymbol{x}) \delta^{3}\left(\boldsymbol{x}-\boldsymbol{x}^{\prime}\right)\right] \delta\left(t-t^{\prime}\right)
\end{aligned}
$$

for carrier diffusion, and

$$
\begin{aligned}
& \left\langle\theta_{D}(\boldsymbol{x}, t) \theta_{D}\left(\boldsymbol{x}^{\prime}, t^{\prime}\right)\right\rangle \\
& \quad=\frac{2 D_{T} k_{B} T_{0}^{2}}{k} \nabla_{\boldsymbol{x}} \cdot \nabla_{\boldsymbol{x}^{\prime}} \delta^{3}\left(\boldsymbol{x}-\boldsymbol{x}^{\prime}\right) \delta\left(t-t^{\prime}\right)
\end{aligned}
$$


for thermal diffusion where $\kappa$ is the specific heat per unit volume and $k_{B}$ is Boltzmann's constant.

We can then split $\Xi=\xi_{D}+\xi_{E}, \Theta=\theta_{D}+\theta_{E}$ into diffusionrelated and particle-related fluctuation terms; the particle terms, after normalizing to number rates, give the following correlations $[15]$ :

$$
\left\langle\Delta_{i}(t) \Delta_{i}\left(t^{\prime}\right)\right\rangle=\left\langle\Delta_{r}(t) \Delta_{r}\left(t^{\prime}\right)\right\rangle=\left(\frac{2 \hbar \omega_{m}^{2}}{\epsilon V A_{0}}\right)^{2} R_{0} \delta\left(t-t^{\prime}\right)
$$

where $R_{0}$ is the sum of all rates that change photon number.

$$
\left\langle\xi_{E}(\boldsymbol{x}, t) \xi_{E}\left(\boldsymbol{x}^{\prime}, t^{\prime}\right)\right\rangle=R_{2}(\boldsymbol{x}) \delta^{3}\left(\boldsymbol{x}-\boldsymbol{x}^{\prime}\right) \delta\left(t-t^{\prime}\right)
$$

where $R_{2}$ is the rate per unit volume of events that change carrier density.

$$
\left\langle\theta_{E}(\boldsymbol{x}, t) \theta_{E}\left(\boldsymbol{x}^{\prime}, t^{\prime}\right)\right\rangle=\left(\frac{E_{\mathrm{NR}}}{\kappa}\right)^{2} R_{3}(\boldsymbol{x}) \delta^{3}\left(\boldsymbol{x}-\boldsymbol{x}^{\prime}\right) \delta\left(t-t^{\prime}\right)
$$

where $E_{\mathrm{NR}}$ is the mean thermal energy dissipated in a nonradiative (or absorptive) event and $R_{3}$ is the event rate per unit volume.

In addition, there are several nonzero off-diagonal correlations, reflecting the fact that many events change more than one system at a time. They are as follows:

$$
\left\langle\xi_{E}(\boldsymbol{x}, t) \Delta_{i}\left(t^{\prime}\right)\right\rangle=\left(\frac{2 \hbar \omega_{m}^{2}}{\epsilon V A_{0}}\right) R_{1}(\boldsymbol{x}) \delta\left(t-t^{\prime}\right)
$$

where $R_{1}(\boldsymbol{x})$ is the rate of stimulated transitions.

$$
\left\langle\theta_{E}(\boldsymbol{x}, t) \Delta_{i}\left(t^{\prime}\right)\right\rangle=\left(\frac{2 \hbar^{2} \omega_{m}^{3}}{\epsilon V A_{0} \kappa}\right) R_{4}(\boldsymbol{x}) \delta\left(t-t^{\prime}\right)
$$

where $R_{4}$ is the rate of heat-generating absorptions.

$$
\left\langle\theta_{E}(\boldsymbol{x}, t) \xi_{E}\left(\boldsymbol{x}^{\prime}, t^{\prime}\right)\right\rangle=\left(\frac{E_{\mathrm{NR}}}{\kappa}\right) R_{5}(\boldsymbol{x}) \delta^{3}\left(\boldsymbol{x}-\boldsymbol{x}^{\prime}\right) \delta\left(t-t^{\prime}\right)
$$

where $R_{5}$ is the rate of nonradiative carrier recombination. All other possible off-diagonal terms are negligible or zero, including $\left\langle\Delta_{i} \Delta_{r}\right\rangle[15]$.

\section{B. Langevin Sources in the Transform Plane}

Equations (28) and (29) are in terms of transformed variables, so it is convenient to cast (33)-(40) in the same manner, particularly since we will eventually be interested in spectral functions $W_{f g}(\Omega)$ which are themselves transformed quantities. Mathematical problems arise when one attempts to take the Fourier transform of a stationary signal, however. (While it is always possible to solve the equations by Laplace transform, the complications due to the initial conditions make the calculation somewhat tedious.) To be rigorous, one must use finitedomain Fourier transforms defined as follows:

$$
\begin{aligned}
& \tilde{f}_{T}(\omega) \equiv \int_{-T / 2}^{+T / 2} d t f(t) e^{-j \omega t}, \\
& \tilde{g}_{T}(\omega) \equiv \int_{-T / 2}^{+T / 2} d t g(t) e^{-j \omega t} .
\end{aligned}
$$

Then, to calculate the spectral term defined by the WeinerKhintchine relations as

$$
W_{f g}(\Omega) \equiv \int d \tau\langle f(t) g(t+\tau)\rangle e^{-j \Omega t}
$$

In terms of the transforms (41),

$$
W_{f g}(\Omega)=\lim _{T \rightarrow \infty} \frac{\left\langle\breve{f}_{T}(\Omega) \tilde{g}_{T}(-\Omega)\right\rangle}{T} .
$$

Strictly speaking, the relations which make the Fourier transform useful (e.g., transformation of differential operators) are not exact so long as the object of the transform is finite at the limits of integration. However, as $T \rightarrow \infty$, the results of the spectral calculation become valid. In deference to our use of properties of the infinite-domain transform, we will drop the $\widetilde{f}_{T}$ notation and continue to use $\widetilde{f}$.

We are also interested in spatial Fourier transforms of the Langevin sources in the same way that we defined transforms for the dynamical variables in (15) and (16). The calculations are somewhat simplified if we make the assumption that all carrier rates are uniform over the active region. The transformed (33)-(40) are

$$
\widetilde{\widetilde{W}}_{\xi_{D} \xi_{D}}\left(\boldsymbol{n}, \boldsymbol{n}^{\prime}, \Omega\right) \approx \frac{D_{E} \gamma(\boldsymbol{n})}{4} \delta_{\boldsymbol{n}, \boldsymbol{n}^{\prime}} \bar{M}
$$

where

$$
\begin{aligned}
& \bar{M} \equiv \int_{V_{A}} d^{3} \boldsymbol{x}\left[-\nabla^{2} N_{0}(\boldsymbol{x})\right] \\
& \widetilde{\tilde{W}}_{\theta_{D^{\theta} D}}\left(\boldsymbol{k}, \boldsymbol{k}^{\prime}, \Omega\right)=\frac{-2 D_{T} k_{B} T_{0}^{2}}{\kappa} \boldsymbol{k} \cdot \boldsymbol{k}^{\prime}(2 \pi)^{3} \delta^{3}\left(\boldsymbol{k}+\boldsymbol{k}^{\prime}\right) \\
& W_{\Delta_{i} \Delta_{i}}(\Omega)=W_{\Delta_{r} \Delta_{r}}(\Omega)=\left(\frac{2 \hbar \omega_{m}^{2}}{\epsilon V A_{0}}\right)^{2} R_{0} \\
& \widetilde{\widetilde{W}}_{\xi_{E} \xi_{E}}\left(\boldsymbol{n}, \boldsymbol{n}^{\prime}, \Omega\right)=\frac{\gamma(\boldsymbol{n})}{8} V_{A} R_{2} \delta_{\boldsymbol{n}, \boldsymbol{n}^{\prime}} \\
& \widetilde{\tilde{W}}_{\theta_{E^{\theta} E}}\left(\boldsymbol{k}, \boldsymbol{k}^{\prime}, \Omega\right)=\left(\frac{E_{\mathrm{NR}}}{\kappa}\right)^{2} \widetilde{R}_{3}\left(\boldsymbol{k}+\boldsymbol{k}^{\prime}\right) \\
& \tilde{W}_{\Delta_{i} \xi E}(\boldsymbol{n}, \Omega)=\tilde{W}_{\xi_{E} \Delta_{i}}(\boldsymbol{n}, \Omega)=\left(\frac{2 \hbar \omega_{m}^{2}}{\epsilon V A_{0}}\right) R_{1} \delta_{\boldsymbol{n}, 0} \\
& \widetilde{W}_{\Delta_{i} \theta_{E}}(\boldsymbol{k}, \Omega)=\tilde{W}_{\theta_{E} \Delta_{i}}(\boldsymbol{k}, \Omega)=\left(\frac{2 \tilde{h}^{2} \omega_{m}^{3}}{\epsilon V A_{0} K}\right) \widetilde{R}_{4}(\boldsymbol{k}) \\
& \widetilde{\widetilde{W}}_{\theta_{E} \xi_{E}}(\boldsymbol{k}, \boldsymbol{n}, \Omega)=\widetilde{\widetilde{W}}_{\xi_{E} \theta_{E}}(\boldsymbol{k}, \boldsymbol{n}, \Omega) \\
& =\left(\frac{E_{\mathrm{NR}}}{\kappa}\right) R_{5} \frac{V_{A}}{8} \prod_{i=1}^{3}\left[e^{\left(-i q_{i}(n) S_{i}\right) / 2}\right. \\
& \cdot \operatorname{sinc} \frac{\left(k_{i}+q_{i}(\boldsymbol{n})\right) S_{i}}{2}+e^{\left(+i q_{i}(\boldsymbol{n}) S_{i}\right) / 2} \\
& \left.\cdot \operatorname{sinc} \frac{\left(k_{i}-q_{i}(n)\right) S_{i}}{2}\right]
\end{aligned}
$$

where $\operatorname{sinc}(x) \equiv \sin (x) / x$ and $\delta_{n, n^{\prime}}$ is the Kronecker delta. 
None of the Langevin spectra have any frequency dependence, which is as it should be; the nature of the Langevin approach is that the sources are Markoffian [16] and therefore, white.

\section{Fluctuation Spectra}

\section{A. Relative Intensity and Frequency Fluctuation Spectra}

The relative intensity fluctuation spectrum is defined as

$$
W_{\rho \rho}(\Omega) \equiv \lim _{T \rightarrow \infty} \frac{\langle\tilde{\rho}(\Omega) \tilde{\rho}(-\Omega)\rangle}{T}
$$

while the frequency fluctuation spectrum is

$$
\begin{aligned}
W_{\Delta \omega}(\Omega) & \equiv \lim _{T \rightarrow \infty} \frac{\langle\tilde{\varphi}(\Omega) \tilde{\varphi}(-\Omega)\rangle}{T} \\
& =\lim _{T \rightarrow \infty} \frac{\langle[j \Omega \tilde{\varphi}(\Omega)][-j \Omega \tilde{\varphi}(\Omega)]\rangle}{T} .
\end{aligned}
$$

We make use of (28) and (29) for $\tilde{\rho}$ and $j \Omega \tilde{\varphi}$, and relations (44)-(51) for the Langevin spectra. The tedium of the algebra is somewhat relieved if we note that the off-diagonal driving terms (e.g., $W_{\Delta_{i} \xi_{E}}$ ) are of importance only near a crossing of the associated diagonal spectra (e.g., $W_{\Delta_{i} \Delta_{i}}, W_{\xi_{E} \xi_{E}}$ ); while a full solution must contain the off-diagonal terms, we lose very little by ignoring them for the rest of the analysis. Define $\alpha_{N} \equiv \zeta_{r} / \zeta_{i}$ and $\alpha_{T} \equiv \bar{\eta}_{r} / \bar{\eta}_{i}$; the relative intensity fluctuation spectrum including photon, carrier, and temperature noise sources, is

$$
\begin{aligned}
& W_{\rho \rho}(\Omega)=|R(\Omega)|^{2}\left(\frac{\omega_{m}}{2 \mu^{2} V}\right)^{2} \\
& \left\{\sum_{\boldsymbol{n}} \Lambda^{2}(\boldsymbol{n}) \zeta_{i}^{2} \frac{\gamma(\boldsymbol{n})}{8} \frac{2 D_{E} \bar{M}}{\Omega^{2}+\left(D_{E} q^{2}(\boldsymbol{n})+\frac{1}{\tau_{R}}\right)^{2}}\right. \\
& +\sum_{\boldsymbol{n}} \Lambda^{2}(\boldsymbol{n}) \zeta_{i}^{2} \frac{\gamma(\boldsymbol{n})}{8} \frac{V_{A} R_{2}}{\Omega^{2}+\left(D_{E} q^{2}(\boldsymbol{n})+\frac{1}{\tau_{R}}\right)^{2}} \\
& +\int d^{3} \boldsymbol{k} d^{3} \boldsymbol{k}^{\prime} \Gamma(\boldsymbol{k}) \Gamma\left(\boldsymbol{k}^{\prime}\right) \bar{\eta}_{i}^{2} \\
& \frac{\frac{-2 D_{T} k_{B} T_{0}^{2}}{k} \boldsymbol{k} \cdot \boldsymbol{k}^{\prime}(2 \pi)^{3} \delta^{3}\left(\boldsymbol{k}+\boldsymbol{k}^{\prime}\right)}{\left(j \Omega+D_{T} k^{2}\right)\left(-j \Omega+D_{T} k^{\prime 2}\right)} \\
& +\int d^{3} \boldsymbol{k} d^{3} \boldsymbol{k}^{\prime} \Gamma(\boldsymbol{k}) \Gamma\left(\boldsymbol{k}^{\prime}\right) \bar{\eta}_{i}^{2} \\
& \left.\cdot \frac{\left(\frac{E_{\mathrm{NR}}}{k}\right)^{2} \tilde{R}_{3}\left(\boldsymbol{k}+\boldsymbol{k}^{\prime}\right)}{\left(j \Omega+D_{T} k^{2}\right)\left(-j \Omega+D_{T} k^{\prime 2}\right)}\right\} \\
& +|R(\Omega)|^{2}\left(\frac{\hbar \hbar \omega_{m}}{\epsilon V A_{0}^{2}}\right)^{2} R_{0} .
\end{aligned}
$$

The frequency fluctuation spectrum is

$$
\begin{aligned}
& W_{\Delta \omega}(\Omega)=\alpha_{n}^{2} \Omega^{2}|R(\Omega)|^{2}\left(\frac{\omega_{m}}{2 \mu^{2} V}\right)^{2} \\
& \left\{\sum_{\boldsymbol{n}} \Lambda^{2}(\boldsymbol{n}) \zeta_{i}^{2} \frac{\gamma(\boldsymbol{n})}{8} \frac{2 D_{E} \bar{M}}{\Omega^{2}+\left(D_{E} q^{2}(\boldsymbol{n})+\frac{1}{\tau_{R}}\right)^{2}}\right. \\
& +\sum_{\boldsymbol{n}} \Lambda^{2}(\boldsymbol{n}) \zeta_{i}^{2} \frac{\gamma(\boldsymbol{n})}{8} \frac{V_{A} R^{2}}{\Omega^{2}+\left(D_{E} q^{2}(\boldsymbol{n})+-\right.} \\
& +\left|\alpha_{n}(1-j \Omega R)-\alpha_{T}\right|^{2}\left(\frac{\omega_{m}}{2 \mu^{2} V}\right)^{2} \\
& \left\{\int d^{3} \boldsymbol{k} d^{3} \boldsymbol{k}^{\prime} \Gamma(\boldsymbol{k}) \Gamma\left(\boldsymbol{k}^{\prime}\right) \vec{\eta}_{i}^{2}\right. \\
& \frac{\frac{-2 D_{t} k_{B} T_{0}^{2}}{\kappa} \boldsymbol{k} \cdot \boldsymbol{k}^{\prime}(2 \pi)^{3} \delta^{3}\left(\boldsymbol{k}+\boldsymbol{k}^{\prime}\right)}{\left(j \Omega+D_{T} k^{2}\right)\left(-j \Omega+D_{T} k^{\prime 2}\right)} \\
& +\int d^{3} \boldsymbol{k} d^{3} \boldsymbol{k}^{\prime} \Gamma(\boldsymbol{k}) \Gamma\left(\boldsymbol{k}^{\prime}\right) \bar{\eta}_{i}^{2} \\
& \left.\left.\frac{\left(\frac{E_{\mathrm{NR}}}{\kappa}\right) \tilde{R}_{3}\left(\boldsymbol{k}+\boldsymbol{k}^{\prime}\right)}{\left(j \Omega+D_{T} k^{2}\right)\left(-j \Omega+D_{T} k^{\prime 2}\right)}\right)\right\} \\
& +\left[1+\alpha_{N}^{2}|1-j \Omega R|^{2}\right]\left(\frac{\hbar \omega_{m}}{\epsilon V A_{0}^{2}}\right)^{2} R_{0} .
\end{aligned}
$$

Equations (54a)-(54e) and (55a)-(55e) are plotted in Figs. 3(a)-(e) and 4(a)-(e), respectively, for a typical laser with parameters listed in Table I. There are several features of interest in the equations and graphs. Equations (54e) and (55e) are the familiar expressions one derives using the standard volume-averaged rate equation analysis [15]. They contain a relaxation resonance in the intensity noise [10] and the same resonance in the frequency noise, along with the linewidth enhancement factor $\alpha_{N}$ [9], [15]. The remaining expressions are those that are lost in the volume-averaged treatment. We can see from the graphs that both the diffusiondriven carrier fluctuations, (54a) and (55a), and the spontaneous emission-driven carrier fluctuations (54b), (55b) make negligible contributions to the intensity noise and frequency fluctuation spectra [Figs. 3(b) and 4(b)]. Closer examination of the summations in (54b) and (55b) will show why this is so. Each term makes a contribution with a corner frequency of $\Omega_{0}=D_{E} q^{2}(\boldsymbol{n})+1 / \tau_{R}$; this is an indication of diffusion damping above $\Omega_{0}$. Below $\Omega_{0}$, where diffusion is less effective, however, the carrier lifetime $\tau_{R}$ is responsible for damping the perturbation. In other words, spatial perturbations to the carrier density are damped out before they can make a substantial low-frequency contribution to the noise spectrum. 


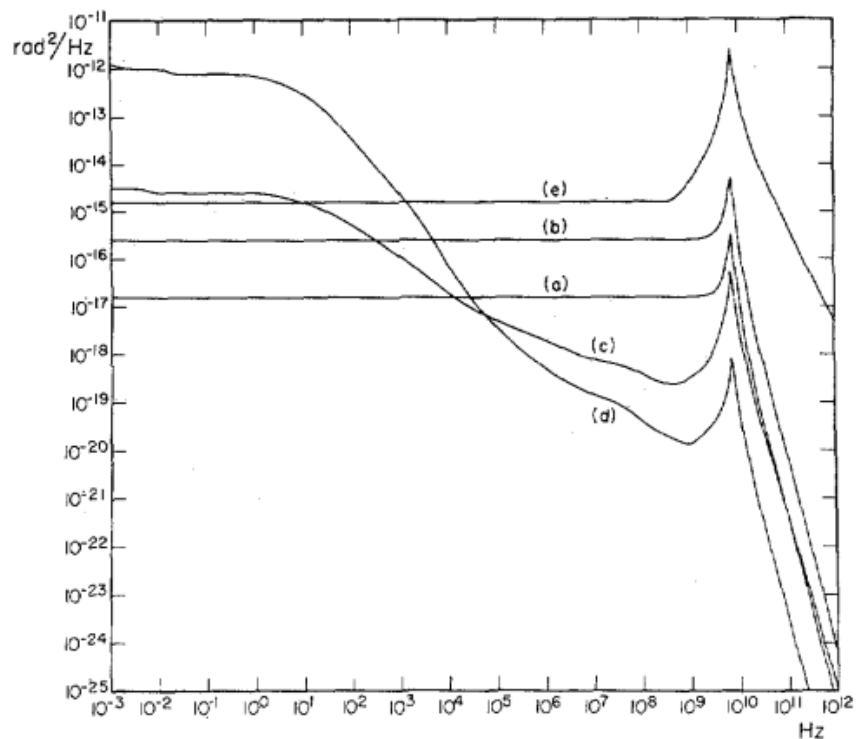

Fig. 3. Noise terms in the intensity noise spectrum [(54a)-(54e)] for the parameters listed in Table I. (a) Carrier diffusion. (b) Carrier creation/annihilation. (c) Temperature diffusion. (d) Nonradiative recombination/absorption temperature fluctuations. (e) Spontaneous emission.

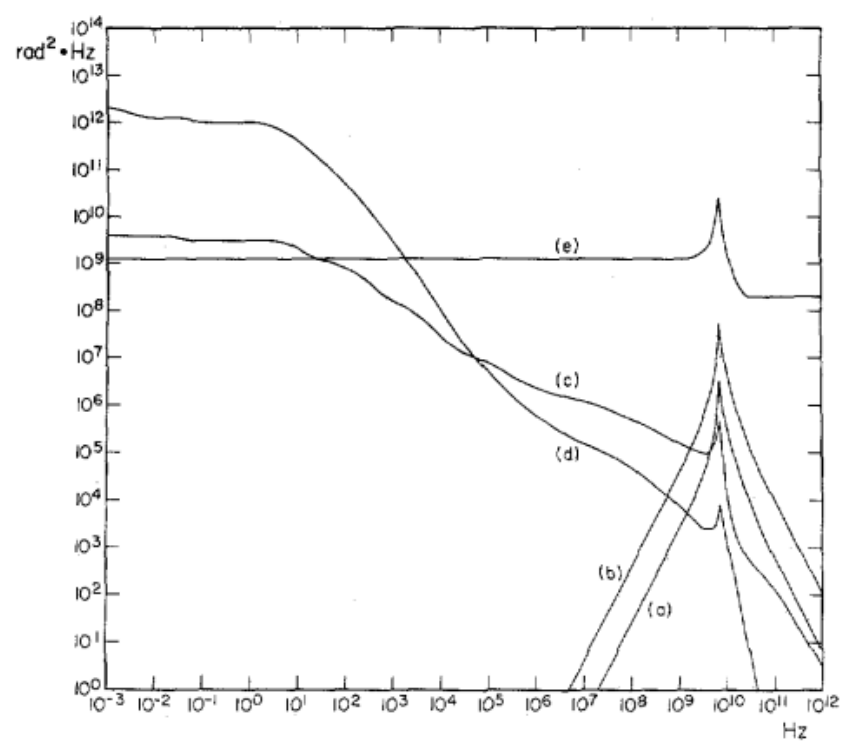

Fig. 4. Noise terms in the frequency fluctuation spectrum [ $(55 \mathrm{a})-(55 \mathrm{e})]$ for the parameters listed in Table I. (a) Carrier diffusion. (b) Carrier creation/annihilation. (c) Temperature diffusion. (d) Nonradiative recombination/absorption temperature fluctuations. (e) Spontaneous emission.

Additional carrier noise sources (for example, noise in the pump current) can be similarly treated by inclusion into the $\Xi$ Langevin source.

The fluctuations due to temperature diffusion $(c)$ are, by themselves, greater than the noise produced by spontaneous emission (e); they are, however, themselves masked by the noise from event-driven thermal fluctuations $(d)$. Two major contributors to this noise source are nonradiative recombination and optical absorption. These produce delta-like impulses of heat (Voss and Clarke's $P$-source revived [13]). Since there is no thermal lifetime (the equivalent to $1 / \tau_{R}$ ), the tempera-
TABLE I

Laser Parameters Assumed in the Graphs of Figs. 3 and 4

$\begin{aligned} \alpha_{N} & =-2.2 \\ \alpha_{T} & =-0.90 \\ \omega_{m} & =2.2 \cdot 10^{15} \mathrm{rad} / \mathrm{s}^{-1} \\ \omega_{S} & =10^{9} \mathrm{~s}^{-1} \\ N_{0} & =10^{18} \mathrm{~cm}^{-3} \\ \tau_{p} & =2 \cdot 10^{-12} \mathrm{~s} \\ S_{1} & =0.2 \mu \mathrm{m} \\ S_{2} & =2.0 \mu \mathrm{m} \\ S_{3} & =200 \mu \mathrm{m} \\ V & =V A=8.0 \cdot 10^{-11} \mathrm{~cm}^{3} \\ \zeta_{i} & =7.3 \cdot 10^{-21} \mathrm{~cm}^{3} \\ \eta_{i} & =4.7 \cdot 10^{-4} \mathrm{~K}^{-1} \\ P_{0} & =5.0 \cdot 10^{-3} \mathrm{~W} \\ \tau_{R} & =1.9 \cdot 10^{-10} \mathrm{~s} \\ \omega_{R} & =4.6 \cdot 10^{10} \mathrm{rad} / \mathrm{s}^{-1} \\ R_{0} & =4.2 \cdot 10^{17} \mathrm{~s}^{-1} \\ R_{2} & =5.3 \cdot 10^{27} \mathrm{~cm}^{-3} \cdot \mathrm{s}^{-1} \\ R_{3} & =1.3 \cdot 10^{27} \mathrm{~cm}^{-3} \cdot \mathrm{s}^{-1} \\ \bar{M} & =5.0 \cdot 10^{16} \mathrm{~cm}^{-2} \\ E_{\mathrm{NR}} & =2.3 \cdot 10^{-19} \mathrm{~J} \\ \kappa & =1.9 \mathrm{~J} \cdot \mathrm{K}^{-1} \cdot \mathrm{cm}^{-3} \\ D_{E} & =220 \mathrm{~cm}^{2} / \mathrm{s}^{-1} \\ D_{T} & =0.24 \mathrm{~cm}^{2} / \mathrm{s}^{-1} \\ & \end{aligned}$

ture fluctuations extend to verv low frequency before leveling off due to the finite volume of the lasing region. The fluctuations couple to the amplitude via gain dependence upon temperature, and to the phase via the refractive index dependence. It is instructive to compare the photon and temperature noise in the intensity spectrum with the corresponding noise in the frequency spectrum. The noise terms are substantially the same (aside from the leading $|R(\Omega)|^{2}$ ), but the photon noise in the frequency spectrum is enhanced by a factor $1+\alpha_{N}^{2}$ over its counterpart in the intensity spectrum, and exhibits a resonance. This is due to the amplitude-phase coupling through carrier dynamics [9], [11], [15] and was observed by Fleming and Mooradian [2]. The temperature fluctuation-driven noise in the frequency spectrum is enhanced by a factor $\left(\alpha_{N}-\alpha_{T}\right)^{2}$. Here, $\alpha_{T}$ is a new coupling parameter; it represents amplitudephase fluctuations coupled through the temperature dependence of gain and index. $\alpha_{N}$ has been measured and calculated [18], [19] to be approximately -2 to -4 , while $\alpha_{T}$ is on the order of -1 . The frequency dependence of this noise source is of the form $1 / f^{\alpha}, \alpha \approx 1$ for several decades, which is in agreement with experimental measurements from several sources $[5]-[7],[20],[25]-[27]$. The noise should exhibit the relaxation resonance before falling off as $1 / f^{2}$ at high frequency; this behavior, however, is in practice masked by other processes. 


\section{B. Field Spectrum and Linewidth}

The field spectrum of the laser diode is given by

$$
\begin{aligned}
& \left\langle E_{n}(t+\tau) E_{n}^{*}(t)\right\rangle \\
& \quad=A_{0}^{2}\left\langle(1+\rho(t+\tau)) e^{j \varphi(t+\tau)}(1+\rho(t)) e^{j \varphi(t)}\right\rangle e^{j \omega_{m} \tau} .
\end{aligned}
$$

If amplitude fluctuations are negligible or suppressed in the measurement process (as, for example, was done in [20]) then the field spectrum can be put in terms of the frequency fluctuation spectrum [22] as

$$
\begin{aligned}
W_{\epsilon}\left(\omega+\omega_{m}\right)= & \frac{1}{2} A_{0}^{2} \operatorname{Re}^{\prime} \int_{-\infty}^{+\infty} d \tau e^{-j \omega \tau} \\
& \cdot \exp \left[\frac{-\tau^{2}}{2 \pi} \int_{0}^{\infty} d \Omega W_{\Delta \omega}(\Omega)\left(\frac{\sin \frac{\Omega \tau}{2}}{\frac{\Omega \tau}{2}}\right)^{2}\right]
\end{aligned}
$$

where $\omega$ is the deviation from the lasing frequency $\omega_{m}$. In the case where $W_{\Delta \omega}$ is a sum of several terms, then $W_{\epsilon}$ will be the convolution of the individual $W_{\epsilon}$ 's calculated from each of the terms of $W_{\Delta \omega}$. So, for example, a dc component of $W_{\Delta \omega}$ results in a Lorentzian line

$$
W_{\epsilon}\left(\omega+\omega_{m}\right)=\frac{A_{0}^{2}}{4 \pi} \frac{W_{\Delta \omega}^{\mathrm{DC}}(0)}{\omega^{2}+\left(\frac{W_{\Delta \omega}^{\mathrm{DC}}(0)}{2}\right)^{2}} .
$$

This has a linewidth of $W_{\Delta \omega}^{\mathrm{DC}}(0)$, namely $\left(1+\alpha_{N}^{2}\right)\left(\hbar \omega / \epsilon V A_{0}^{2}\right) R_{0}$, the enhanced Schawlow-Townes linewidth. To include the contribution of the recombination noise, we note from Fig. 3 that the spectrum at low frequency is approximately $1 / f$-like with a corner frequency near $1 \mathrm{~Hz}$ : we model it as

$$
W_{\Delta \omega}^{\mathrm{NR}}(\Omega)=\frac{\omega_{0}^{2}}{\Omega+\omega_{1}} .
$$

This leads to an approximate lineshape of

$$
\frac{1}{2 \pi} \int_{-\infty}^{+\infty} d \tau e^{-j \omega \tau} \exp \left\{\frac{-\tau^{2} \omega_{0}^{2}}{2 \pi} \ln \left(1+\frac{2}{\omega_{1}|\tau|}\right)\right\} .
$$

This is not an easy Fourier transform to evaluate, but we can pick out the contribution to linewidth by exploiting the Fourier uncertainty relationship, that

$$
\Delta \tau \Delta \omega \approx 2
$$

so that the linewidth $\Delta \omega$ satisfies the implicit relation

$$
\Delta \omega \approx \omega_{0}\left[\ln \left(\frac{\Delta \omega}{\omega_{1}}\right)\right]^{1 / 2}
$$

For the laser with parameters given in Fig. 3, we have $\omega_{0}^{2} \approx$ $3 \cdot 10^{12} \mathrm{rad}^{2} / \mathrm{s}^{2}, \omega_{1} \approx 3 \mathrm{rad}^{2} / \mathrm{s}$ which gives $\Delta \omega \approx 6.6 \cdot 10^{6}$ $\mathrm{rad} / \mathrm{s}$ and $\Delta f \approx 1.1 \mathrm{MHz}$. This is comparable to the powerindependent linewidth observed by Welford and Mooradian [3], [24] and Kikuchi and Okoshi [25]. While carrier fluc- tuations are strongly damped by the carrier lifetime $\tau_{R}$ at low frequency, and, as can be seen from Fig. 4, make minimal contributions to the frequency fluctuation spectrum (and hence, linewidth), temperature-induced index/gain variations are not similarly damped. A primary source for these fluctuations is nonradiative recombination, which is a function of carrier density, and therefore is constant above threshold. Hence, the resulting contribution to linewidth is powerindependent.

\section{CONCLUSION}

In this paper we have derived the fluctuation spectra of a single-mode semiconductor laser that result from spatially dependent fluctuations in carrier density and temperature distribution. We have shown that the relaxation resonance which appears in both the intensity noise and frequency fluctuation spectra is damped more effectively than that predicted by spatially independent models; and, that carrier and temperature fluctuations are most important at low frequencies, where some of them mask the flat inverse power spectra reported elsewhere. Carrier fluctuations are damped by the short carrier lifetime; perturbations to the carrier density do not persist long enough to make significant low-frequency contributions, while at higher frequencies they are damped by diffusion. Temperature fluctuations, subject to no such low-frequency damping, play a major role in low-frequency fluctuations. Events which produce isolated rises in temperature (e.g., nonradiative recombination, absorption) give rise to a spectrum that increases at low frequencies, contains decades of $1 / f$-like behavior and levels off below $1 \mathrm{~Hz}$. The frequency spectrum displays an enhancement due to the detuned nature of the gain spectrum; the enhancement factor contains both the carrier-related amplitude-phase coupling coefficient and a new, temperature-related amplitude-phase coupling coefficient. One additional prediction of the theory is a power-independent linewidth comparable to that which has been observed experimentally.

\section{REFERENCES}

[1] T. L. Paoli and J. E. Ripper, "Observations of intrinsic quantum fluctuations in semiconductor lasers," Phys. Rev., vol. 2, pp. 2551-2555, 1970.

[2] M. Fleming and A. Mooradian, "Fundamental line broadening of single mode (GaAl)As diode lasers," Appl. Phys. Lett., vol. 38, pp. $511-513,1981$.

[3] D. Welford and A. Moordian, "Observations of linewidth broadening in $(\mathrm{GaAl}) \mathrm{As}$ diode lasers due to electron number fluctuations," Appl. Phys. Lett., vol. 40, pp. 560-562, 1982.

[4] K. Vahala, C. Harder, and A. Yariv, "Observation of relaxation resonance effects in the field spectrum of semiconductor lasers," Appl. Phys. Lett., vol. 42, pp. 211-213, 1983.

[5] G. Tenchio, "Low-frequency intensity fluctuations of CW DH GaAlAs diode lasers," Electron. Lett., vol. 12, p. 562, 1976.

[6] - " "1/f noise of continuous-wave semiconductor lasers," Electron. Lett., vol. 13, p. 614, 1977.

[7] A. Dandridge and A. B. Tveten, "Phase noise of single-mode diode lasers in interferometer systems," Appl. Phys. Lett., vol. 39, pp. $530-532,1981$.

[8] M. Lax, "Quantum noise VII: The rate equations and amplitude noise in lasers," IEEE J. Quantum Electron., vol. QE-3, pp. 37 $46,1967$.

[9] C. H. Henry, "Theory of the linewidth of semiconductor lasers," IEEE J. Quantum Electron., vol. QE-18, pp. 259-264, 1982. 
[10] D. E. McCumber, "Intensity fluctuations in the output of CW laser oscillators I," Phys. Rev., vol. 141, pp. 306-322, 1966.

[11] K. Vahala and A. Yariv, "Semiclassical theory of noise in semiconductor lasers-Part I," IEEE J. Quantum Electron., vol. QE-19, pp. 1096 1101, 1983.

[12] J. B. Moreno, "Volume-averaged rate equations for planar and disk-cavity lasers," J. Appl. Phys., vol. 48, pp. 4152-4162, 1977.

[13] R. F. Voss and J. Clarke, "Flicker $(1 / f)$ noise: Equilibrium temperature and resistance fluctuations," Phys. Rev. B, vol. 13, pp. $556-573,1976$.

[14] K. M. van Vliet and H. Mehta, "Theory of transport noise in semiconductors," Phys. Stat. Solidi (b), vol. 106, pp. 11-30, 1981.

[15] K. Vahala and A. Yariv, "Semiclassical theory of noise in semiconductor lasers-Part II," IEEE J. Quantum Electron., vol. QE-19, pp. 1102-1109, 1983.

[16] K. M. van Vliet, "Markov approach to density fluctuations due to transport and scattering. I. Mathematical formalism," J. Math. Phys., vol. 12, pp. 1981-2011, 1971.

[17] H. Haug and H. Haken, "Theory of noise in semiconductor laser emission," $Z$ Physik, vol. 204, pp. 262-275, 1967.

[18] C. Harder, K. Vahala, and A. Yariv, "Measurement of the linewidth enhancement factor $\alpha$ of semiconductor lasers," Appl. Phys. Lett., vol. 42, pp. 328-330, 1983.

[19] K. Vahala, L. C. Chiu, S. Margalit, and A. Yariv, "On the linewidth enhancement factor $\alpha$ in semiconductor injection lasers," Appl. Phys. Lett., vol. 42, pp. 631-633, 1983.

[20] F. G. Walther and J. E. Kaufmann, "Characterization of GaAlAs laser diode frequency noise," presented at OFC 1983, New Orleans, LA, paper TUJ5.

[21] T. K. Yee, "Intensity noise of GaAlAs laser diodes at various output powers and frequencies," presented at CLEO 1983, Baltimore, MD, paper TUH3.

[22] K. Vahala and A. Yariv, "Power, frequency, and field spectra for single and multimode semiconductor lasers," unpublished.

[23] G. H. B. Thompson, Physics of Semiconductor Laser Devices. New York: Wiley, 1980, pp. 424-433.

[24] J. Harrison and A. Mooradian, "Spectral characteristics of GaAlAs diode lasers at $1.7 \mathrm{~K}$," Appl. Phys. Lett., vol. 45, pp. 318-320, 1983.

[25] K. Kikuchi and T. Okoshi, "Measurement of spectra of and correlation between FM and AM noises in GalAs lasers," Electron. Lett., vol. 19, pp. 812-813, 1983.
[26] M. J. O'Mahony and I. D. Henning, "Semiconductor laser linewidth broadening due to $1 / f$ carrier noise," Electron Lett., vol. 19, pp. 1000-1001, 1983.

[27] M. Ohtsu and S. Kotajima, "Derivation of the spectral width of a $.8 \mu \mathrm{m} \mathrm{AlGaAs}$ laser considering $1 / f$ noise," Japan. J. Appl. Phys., vol. 23 , pp. $760-764,1984$.

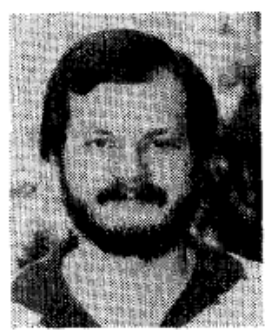

Robert J. Lang (S'83) was born in Dayton, OH on May 4, 1961, and was raised in Atlanta, GA. In 1982 he received the B.S. degree in electrical engineering from the California Institute of Technology, Pasadena, CA. He received the M.S. degree, also in electrical engineering, from Stanford University, Stanford, CA.

Currently, he has been pursuing the Ph.D. degree in applied physics at the California Institute of Technology, where he is investigating dynamic and spectral properties of semiconductor lasers.

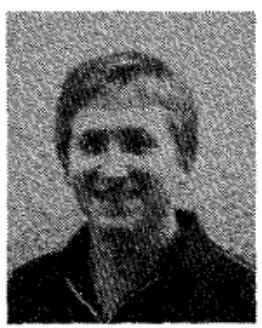

Kerry J. Vahala (S'84) was born in Flint, MI, on February 5, 1959. He received the B.S. degree in applied physics in 1980 and the M.S. degree in electrical engineering in 1981, both from the California Institute of Technology, Pasadena, CA. He completed his doctoral dissertation in the field of semiconductor device physics, also at Caltech.

Dr. Vahala is a member of the Optical Society of America and Tau Beta Pi.

Amnon Yariv (S'56-M'59-F'70), for a photograph and biography, see p. 138 of the February 1985 issue of this JourNAL. 